\title{
Adverse factors on nonenhanced abdominal CT for long-term continuous ambulatory peritoneal dialysis: a comparative study between patients who withdraw from and maintain long-term peritoneal dialysis
}

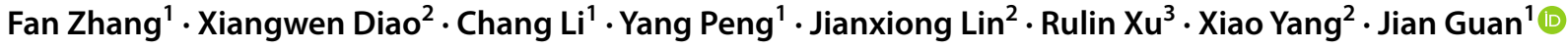

Received: 8 June 2021 / Revised: 3 August 2021 / Accepted: 4 August 2021 / Published online: 14 August 2021

(c) The Author(s), under exclusive licence to Springer Science+Business Media, LLC, part of Springer Nature 2021

\begin{abstract}
Purpose To investigate the imaging features of patients with long-term continuous ambulatory peritoneal dialysis (CAPD) on nonenhanced abdominal CT and to identify adverse factors for long-term CAPD.

Methods A total of 109 patients with less than 5 years of CAPD for peritoneal ultrafiltration failure who switched to hemodialysis (withdrawal group) and 23 patients with more than 10 years of CAPD (long-term group) were retrospectively enrolled. Nonenhanced CT manifestations in both groups were compared, including thickening and calcification of the parietal peritoneum, calcification of the mesangial margin and free margin of the small intestine wall, and calcification of the mesentery and abdominal aorta. A risk stratification model was proposed based on CT manifestations with statistically significant differences.

Results The presence of the following CT findings was significantly different between two groups: extensive thickening of the parietal peritoneum ( $78.9 \%$ vs. $21.7 \%, P<0.01)$; severe calcification of the parietal peritoneum $(60.6 \%$ vs. $8.7 \%, P<0.01)$; calcification of the mesentery ( $32.1 \%$ vs. $4.3 \%, P<0.05)$; and calcification of the free margin of the small intestine wall $(49.5 \%$ vs. $13.0 \%, P<0.05)$. However, there was no significant difference in calcification of the mesangial margin of the small intestine wall (40.3\% vs. $30.4 \%)$ or in abdominal aortic calcification (56.9\% vs. $61.1 \%)(P>0.05)$. The area under the receiver operating characteristic curve (AUC) was 0.906 (sensitivity $87.6 \%$ and specificity $82.6 \%$ ).

Conclusion Extensive thickening of the parietal peritoneum, severe calcification of the parietal peritoneum, and calcification of the mesentery and the free margin of the small intestine wall are adverse factors for long-term CAPD.
\end{abstract}

Keywords Peritoneal dialysis $\cdot$ Long-term $\cdot$ Peritoneum $\cdot$ Small intestine wall $\cdot$ Computed tomography

Fan Zhang and Xiangwen Diao contributed equally to this work, and they are co-first authors

Xiao Yang

yxiao@mail.sysu.edu.cn

Jian Guan

guanj6@mail.sysu.edu.cn

Fan Zhang

zhangf227@mail.sysu.edu.cn

Xiangwen Diao

diaoxw@mail2.sysu.edu.cn

Chang Li

lichang6@mail.sysu.edu.cn

Yang Peng

pengy63@mail.sysu.edu.cn

Jianxiong Lin

ljianx@mail.sysu.edu.cn
Rulin Xu

colin_xu@163.com

1 Department of Radiology, The First Affiliated Hospital, Sun Yat-Sen University, No.58 Zhongshanerlu Road, Guangzhou, Guangdong, People's Republic of China

2 Department of Nephrology, The First Affiliated Hospital, Sun Yat-Sen University, No.58 Zhongshanerlu Road, Guangzhou, Guangdong, People's Republic of China

3 Research Collaboration, Canon Medical Systems (China) Co., LTD, Rm 2906, R\&F Centre, No.10 Huaxia Road, Guangzhou, Guangdong, People's Republic of China 


\section{Abbreviations \\ CAPD Continuous ambulatory peritoneal dialysis \\ PD Peritoneal dialysis \\ ESRD End-stage renal disease \\ ROC Receiver operating characteristic \\ EPS Encapsulating peritoneal sclerosis}

\section{Introduction}

Peritoneal dialysis (PD) is one of the most important methods for renal replacement therapy in patients with end-stage renal disease (ESRD) [1]. PD has some advantages over hemodialysis, including preservation of residual renal function, better quality of life, and lower cost [2]. PD is the most frequent home-based dialysis treatment, especially during the COVID-19 pandemic, as it is not strongly influenced by isolation policies [3]. The peritoneum of PD patients may be gradually injured by hyperosmotic dialysis solution, peritonitis or other reasons. Ultrafiltration failure after peritoneal injury is an important reason that patients withdraw from PD treatment $[4,5]$. The aim of this study was to investigate imaging features on nonenhanced abdominal $\mathrm{CT}$ and to identify adverse factors associated with long-term continuous ambulatory peritoneal dialysis (CAPD).

\section{Materials and methods}

\section{Participants}

This study was approved by the institutional review board. Written informed consent was obtained from all participants. Long-term PD patients with regular medical follow-up were enrolled from September 2003 to December 2019. The inclusion criteria are: (1) diagnosed with ESRD undergoing regular PD longer than 6 months; (2) age above 18 years; (3) baseline examination (before starting PD) by nonenhanced abdominal CT revealed normal appearance of peritoneum, for which all the image indicators (see below in "Image analysis") were negative; and (4) regular follow-up examination by nonenhanced abdominal CT. The exclusion criteria are: (1) acute kidney injury or emergency peritoneal dialysis; (2) kidney transplantation or death due to other complications; (3) patients with secondary hyperparathyroidism and excess D state; (4) incomplete clinical data.

A total of 132 patients were retrospectively enrolled according to the above criteria, including 109 who underwent fewer than 5 years of CAPD for peritoneal ultrafiltration failure and switched to hemodialysis (withdrawal group) and 23 with more than 10 years of CAPD (long-term group). For patients in the withdrawal group, CT images within 3 months before stopping CAPD were collected; CT images in the 10th year of CAPD were collected for the long-term group.

\section{CT data acquisition}

CT examination was performed with a Canon 320-slice CT Aquilion One. Before scanning, the patients fasted for 4-6 h and received dilutions of $800-1000 \mathrm{ml}$ of mannitol orally. Scanning parameters were as follows: tube voltage $120 \mathrm{kV}$, tube current $200 \mathrm{~mA}$, no spacing volume scanning. The scan range was from the diaphragmatic apex to the pelvic floor.

\section{Image analysis}

The imaging manifestations of the two groups on nonenhanced abdominal CT were compared, and the observations included the following six aspects.

(1) Thickness of the parietal peritoneum: extensive thickening was defined as thickness of the parietal peritoneum $\geq$ $3 \mathrm{~mm}$, with a range $\geq 50 \%$ (Fig. 1). The range of peritoneal thickening was determined by the percentage of layers of peritoneal thickening on $\mathrm{CT}$ cross-section to the total number of layers of the abdominal scan. (2) Calcification of the free margin of the small intestine wall (white arrow in Fig. 2). (3) Calcification of the parietal peritoneum: severe calcification was characterized by extensive linear calcification (Fig. 3) or

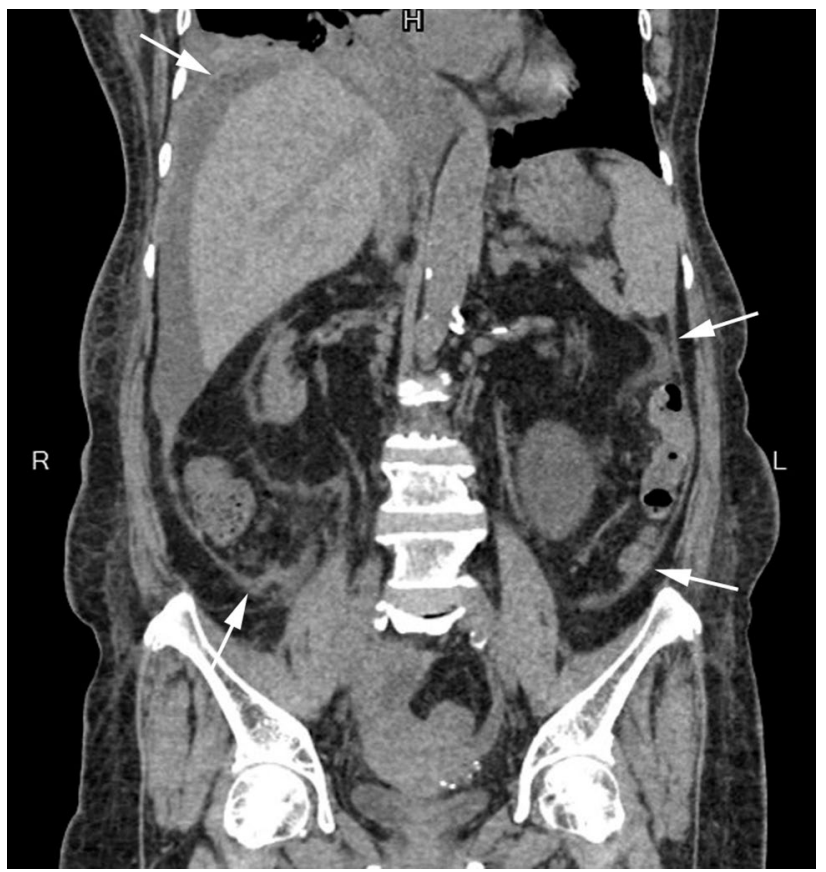

Fig. 1 Glomerulonephritis led to chronic kidney failure in a 63-yearold woman with a history of 11 years of CAPD. The coronal nonenhanced abdominal CT image shows that the parietal peritoneum was extensively thickened (arrows); scored as 1 point 


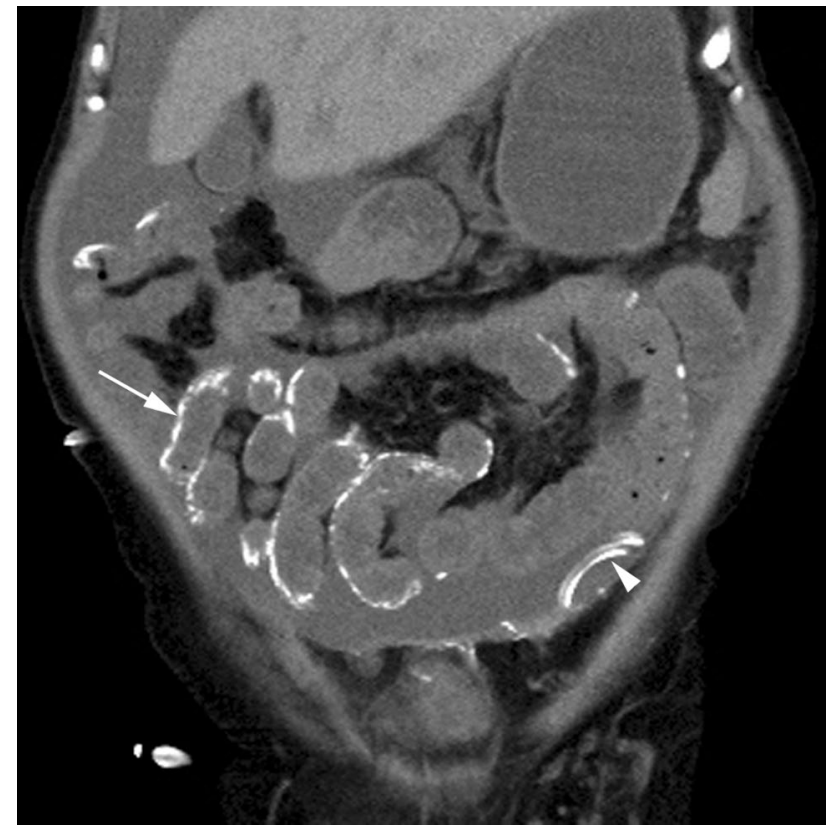

Fig. 2 Chronic kidney failure in a 65-year-old man with a history of 10 years of CAPD. The coronal nonenhanced abdominal CT image shows extensive linear calcification of the free margin of the small intestine wall (arrow); scored as 1 point. Peritoneal dialysis tubes can be seen (arrowhead)

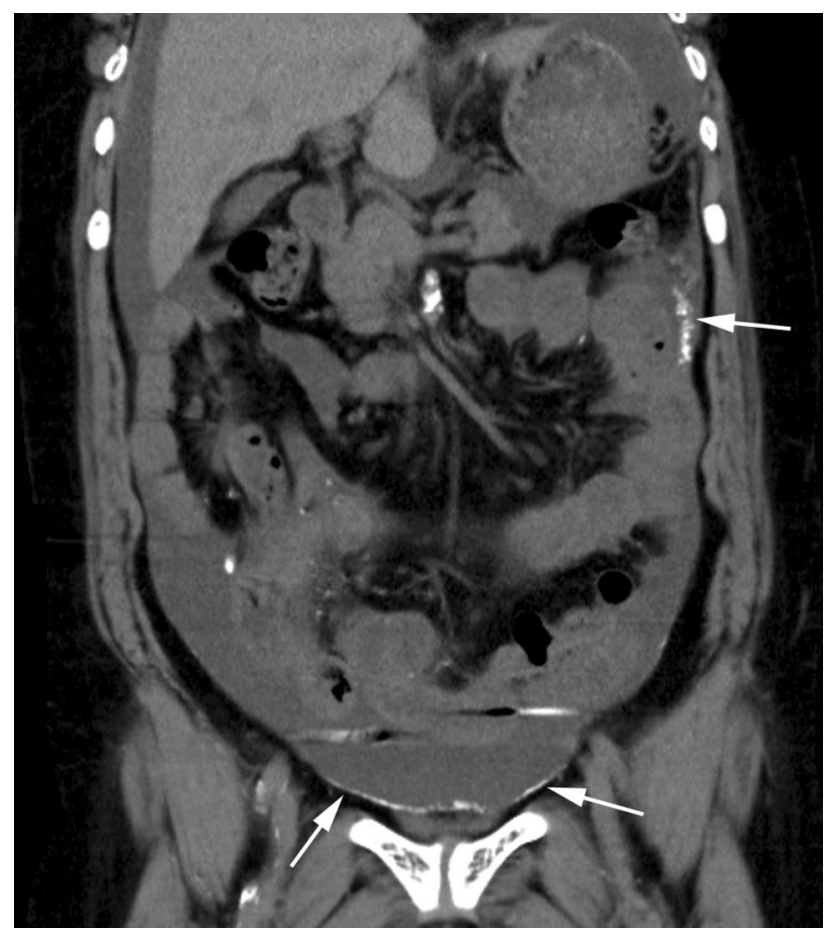

Fig. 3 Diabetic nephropathy led to chronic kidney failure in a 51-year-old man with a history of 3 years of CAPD who switched to hemodialysis because of inadequate dialysis. The coronal nonenhanced abdominal CT image shows that the parietal peritoneum had extensive calcification, such as long linear and short linear lesions (arrows); scored as 2 points

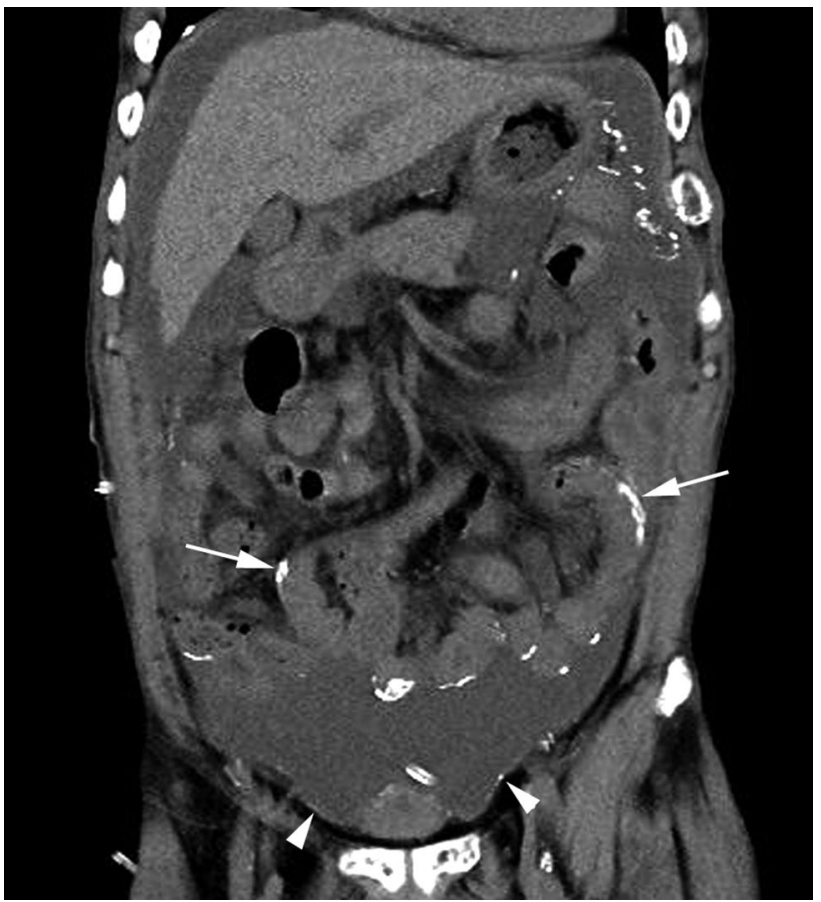

Fig. 4 Chronic renal insufficiency in a 45-year-old woman with a history of 4 years of CAPD who switched to hemodialysis because of an increase in serum creatinine. The coronal nonenhanced abdominal CT image shows calcification of the parietal peritoneum at more than 10 sites (arrowheads), and calcification of the free margin of the mesostenium-free margin can also be seen (arrows); scored as 3 points

scattered calcification involving more than 10 foci (Figs. 4, 5). (4) Calcification of the mesangial margin of the small intestine wall (short white arrow in Fig. 6). (5) Calcification of the mesentery (Fig. 5). (6) Abdominal aortic calcification.

$\mathrm{CT}$ images of all patients were independently qualitatively interpreted by two radiologists (Z.F. with 6 years and P.Y. with 5 years of experience), and discrepancies were resolved via consensus by a senior radiologist (G.J. with 15 years of experience).

\section{Statistical analysis}

SPSS 22.0 statistical software was used. All data are presented as the case (\%) and mean \pm SD or median (25th quartile to 75 th quartile). Independent sample $\mathrm{T}$ tests were used to compare imaging parameters between the two groups, and parameter features with statistically significant differences were then scored according to odds ratios. The image interpretation accuracy was compared by the $\chi^{2}$ test, and consistency between the two readers was analyzed using the kappa test. Two-sided $\mathrm{p}<0.05$ was regarded as significant. 


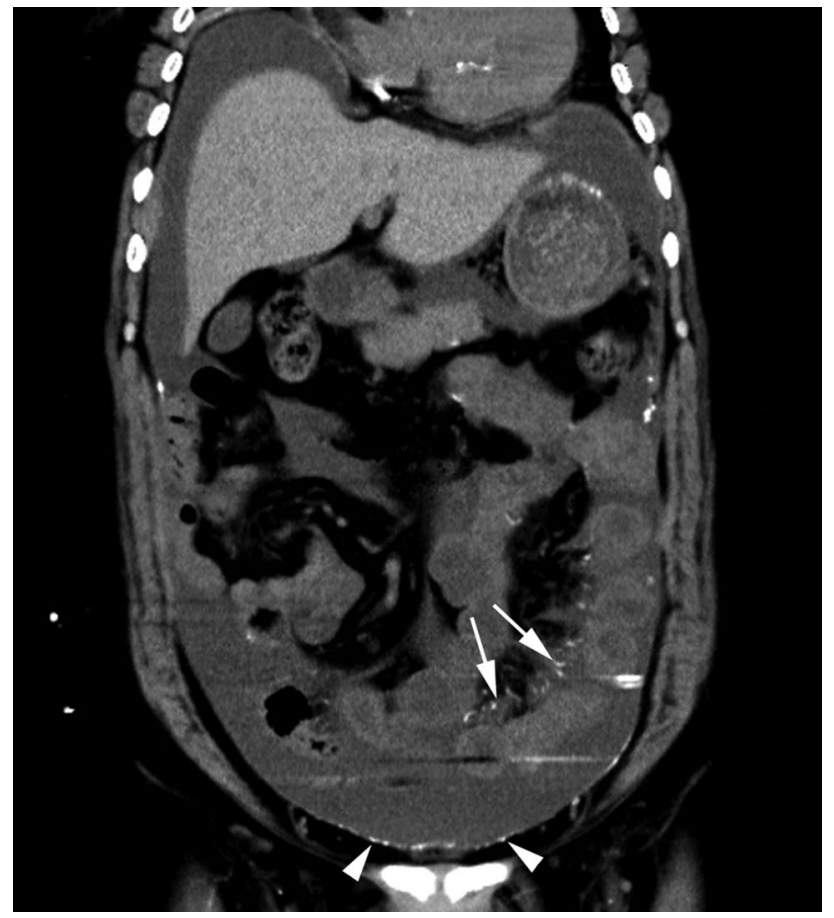

Fig. 5 Hypertensive nephropathy led to chronic kidney failure in a 69-year-old man with a history of 3.5 years of CAPD who switched to hemodialysis because of inadequate dialysis. The coronal nonenhanced abdominal CT image shows multiple places of calcification in the mesentery region (arrows) and more than 10 foci of calcification in the parietal peritoneum (arrowheads); scored as 4 points

\section{Results}

\section{Demographic characteristics of the subjects}

The primary diseases of the two groups included glomerulonephritis, diabetic nephropathy, hypertensive nephropathy, LGA nephropathy and obstructive nephropathy. There were no significant differences in sex, age or percentage of primary disease between the two groups (Table 1).

\section{Imaging manifestations}

Thickening of the parietal peritoneum: Thickening included broadly linear thickening, with higher density. The lesions were mostly located around the liver, at the free margin of the peritoneum on one side or both sides, or in the area where the peritoneum was reversed. The thickness varied from $3 \mathrm{~mm}$ to $5 \mathrm{~mm}$. Compared with the withdrawal group and long-term group, the rate of extensive thickening of the parietal peritoneum was $78.9 \%-21.7 \%$, and the difference was significant $(p=0.031)$.

Calcification of the parietal peritoneum: Calcification was mostly located at the free margin of the parietal peritoneum. Severe calcification was characterized by extensive linear calcifications or scattered calcifications of more than 10 foci, which manifested as short strips, clusters, or lumps. The rate of severe parietal peritoneal calcification between the withdrawal and long-term groups was $60.6 \%$ vs. $8.7 \%$, which was significant $(p<0.001)$.

Calcification of the mesangial margin and the free margin of the small intestine wall: These calcifications were characterized by high-density foci at the mesangial margin or free margin of the small intestine wall, which appeared as speckled, patchy, linear, cluster-shaped lesions. Calcification at the free margin was significantly different between the two groups ( $49.5 \%$ vs. $13.0 \%)(p=0.012)$. However, there was no significant difference between the two groups in calcification of the mesangial margin of the small intestine wall $(40.3 \%$ vs. $30.4 \%)(p=0.074)$.

Calcification of the mesentery: These foci manifested as spotty, patchy, and clustered lesions. Sixty-seven patients in the withdrawal group showed mesenterial calcification. In contrast, only two patients in the long-term group showed a small speckled calcification in the mesentery, and the difference was significant $(p<0.001)$.

Abdominal aortic calcification: Among the 109 patients in the withdrawal group, 62 exhibited abdominal aortic calcification $(56.9 \%)$, whereas $14(61.1 \%)$ of the 23 patients in the long-term group had abdominal aortic calcification. There was no significant difference between the two groups $(p=0.538)$.

Table 1 Comparison of general information between the two groups

\begin{tabular}{lllllllll}
\hline & Age $(\mathrm{y})$ & Male & Female & Glomerulonephritis & $\begin{array}{l}\text { Diabetic nephropa- } \\
\text { thy }\end{array}$ & $\begin{array}{l}\text { Hypertensive } \\
\text { nephropathy }\end{array}$ & $\begin{array}{l}\text { LGA nephropathy Obstructive } \\
\text { nephropathy }\end{array}$ \\
\hline $\begin{array}{l}\text { Withdrawal group } \\
(n=109)\end{array}$ & $(50.3 \pm 19.7)$ & 61 & 48 & $47(43.1 \%)$ & $35(32.1 \%)$ & $17(15.6 \%)$ & $7(6.4 \%)$ & $3(2.8 \%)$ \\
$\begin{array}{c}\text { long-term group } \\
\text { B }(n=23)\end{array}$ & $(53.2 \pm 22.4)$ & 13 & 10 & $11(47.8 \%)$ & $6(26.1 \%)$ & $4(17.5 \%)$ & $1(4.3 \%)$ & $1(4.3 \%)$ \\
$p$ value & 0.350 & 0.183 & 0.688 & 0.442 & 0.150 & 0.313 & 0.205 & 0.352 \\
\hline
\end{tabular}

Data are the mean \pm standard deviation or $\mathrm{n}(\%)$ 
Table 2 Comparison of CT manifestations between the two groups and the score

\begin{tabular}{|c|c|c|c|c|c|}
\hline & $\begin{array}{l}\text { Withdraw group } \\
(n=109)\end{array}$ & $\begin{array}{l}\text { Long-term group } \\
(n=23)\end{array}$ & $p$ value & OR value & Score \\
\hline Extensive thickening of the parietal peritoneum & $86(78.9 \%)$ & $5(21.7 \%)$ & 0.031 & 3.636 & 1 \\
\hline Severe calcification of the parietal peritoneum & $66(60.6 \%)$ & $2(8.7 \%)$ & $<0.001$ & 6.966 & 2 \\
\hline Calcification of the free margin of the small intestine wall & $54(49.5 \%)$ & $3(13.0 \%)$ & 0.012 & 3.808 & 1 \\
\hline Calcification of the mesangial margin of the small intestine wall & $44(40.3 \%)$ & $7(30.4 \%)$ & 0.074 & 1.326 & 0 \\
\hline Calcification of the mesentery & $67(61.5 \%)$ & $2(8.7 \%)$ & $<0.001$ & 7.069 & 2 \\
\hline Abdominal arterial calcification & $62(56.9 \%)$ & $14(61.1 \%)$ & 0.538 & 0.931 & 0 \\
\hline
\end{tabular}

Data are mean \pm standard deviation or $\mathrm{n}(\%)$

Table 3 The score distribution of the two groups

\begin{tabular}{lll}
\hline Total score & Withdraw group $(n=109)$ & $\begin{array}{l}\text { Long-term } \\
\text { group } \\
(n=23)\end{array}$ \\
\hline 0 & 0 & 10 \\
1 & 9 & 8 \\
2 & 27 & 3 \\
3 & 31 & 1 \\
4 & 26 & 1 \\
5 & 14 & 0 \\
6 & 2 & 0 \\
\hline
\end{tabular}

Total score, cumulative score according to the CT observation indexes

A risk stratification model based on the CT manifestations with statistically significant differences was produced, and odds ratios were taken into consideration. The comparison of the CT manifestations between the two groups and the score of the risk stratification model are shown in Table 2. The cumulative score distribution of the two groups is provided in Table 3. The AUC value of the ROC curve was 0.906 , as depicted in Fig. 7. The sensitivity and specificity, 0.876 and 0.826 , respectively, were best at a cutoff value of 2 . The different cases with scores of 1-4 are shown in Figs. 1, 2, 3, 4, 5. According to the $\chi^{2}$ test, there was no significant difference in image interpretation between the two readers $(p<0.05)$. The kappa value was 0.95 , indicating excellent the image interpretation consistency between the two readers.

\section{Discussion}

Peritoneal injury is common in patients on long-term CAPD and is characterized by peritoneal changes in morphology and function, influencing the effect of peritoneal dialysis. In serious cases, the patients even discontinue peritoneal dialysis treatment $[6,7]$. Peritoneal biopsy is the diagnostic method for peritoneal injury. However, it

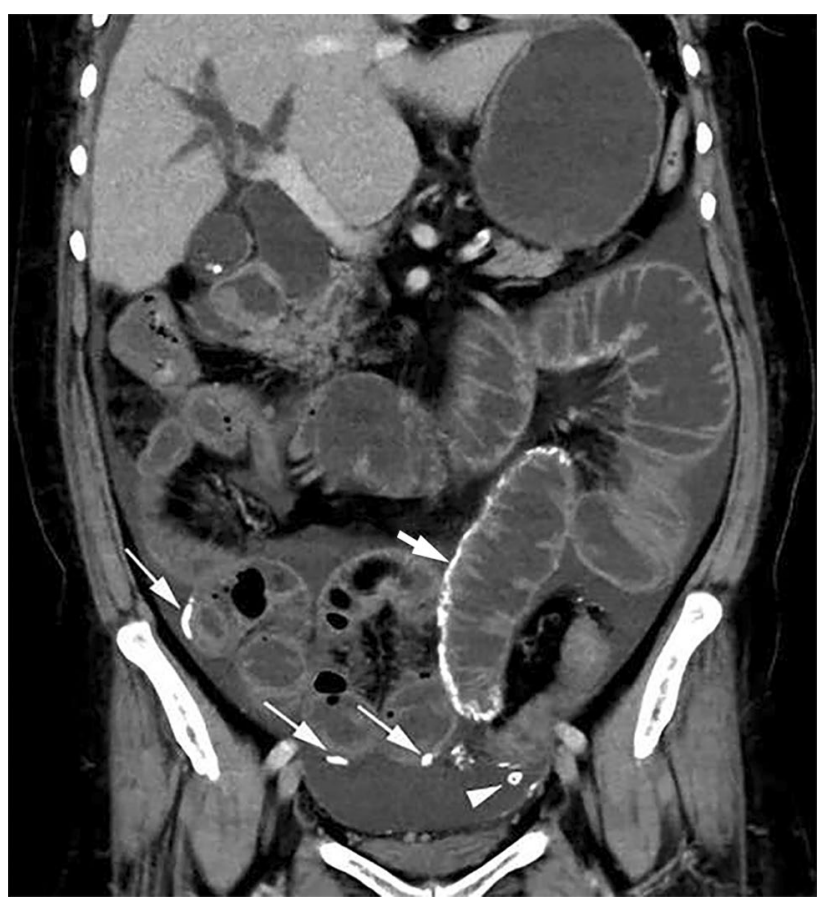

Fig. 6 Calcification of the mesangial margin of the small intestine wall (short white arrow). Calcification of the free margin of the small intestine wall (white arrow). Peritoneal dialysis tubes can be seen (arrowhead)

is difficult to implement in clinical practice for dynamic observation because of the invasive nature [8]. Nonenhanced abdominal CT is a common examination method for PD patients due to its convenience, low cost, and lack of contrast administration [9-11]. In the past, some researchers have analyzed the imaging characteristics of patients with encapsulating peritoneal sclerosis (EPS) by nonenhanced abdominal CT $[12,13]$, which can intuitively reflect peritoneum and abdominal visceral changes and accurately detect calcifications. By comparing the imaging characteristics on nonenhanced abdominal CT of patients who withdraw from or maintain long-term CAPD, we in this study further speculate on the possible mechanism 


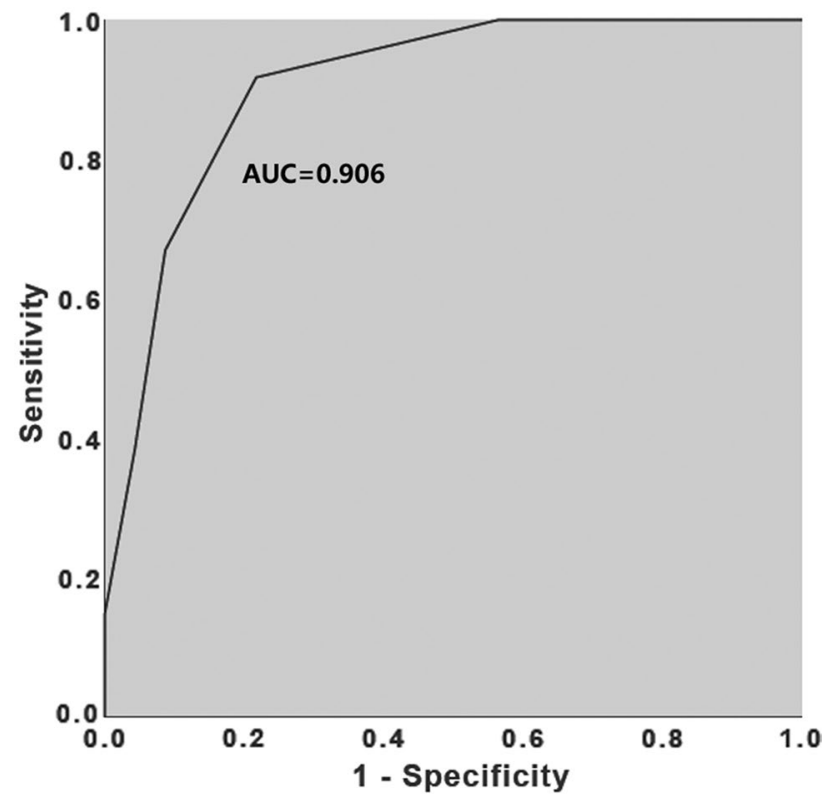

Fig. 7 Receiver operating characteristic (ROC) curve for evaluating the efficacy of peritoneal dialysis: the risk stratification model

of peritoneal changes in PD to identify adverse factors of peritoneal dialysis as early as possible.

PD involves a semipermeable peritoneal membrane. Dialysate removes water via a gradient between the dialysate concentration in the peritoneal cavity and the solute concentration in peritoneal capillaries [14]. The integrity of the peritoneal structure is key to maintaining its function. In this study, abdominal CT findings for two groups of patients with different durations of dialysis were analyzed, and abnormal changes in the parietal peritoneum, small intestine wall and surrounding area were detected in PD patients, consistent with previous reports of abdominal complications [15]. Previous case reports showed that peritoneal calcification may be associated with hyperparathyroidism, excessive vitamin D, repeat peritonitis, and exposure to calcium-containing dialysate and hyperosmotic fluid [16-18]. Overall, the long-term exposure to hyperosmotic dialysate, as well as some inflammatory factors, in the peritoneum of PD patients results in peritoneal thickening or calcification, and the effective surface area and permeability of the peritoneum are reduced [19]. The efficacy of peritoneal dialysis is affected by decreasing peritoneal ultrafiltration capacity and changes in the peritoneal transport state [20]. The results of this study showed no correlation between peritoneal calcification and vascular calcification, consistent with the results of previous studies [21].

Yang et al. [22] analyzed abdominal CT findings of 109 CAPD patients and proposed that parietal peritoneal thickening in these patients is one of the major imaging features of peritoneal injury. Our study is consistent with these findings, and we further showed more extensive thickening and calcification of the parietal peritoneum in the withdrawal group, with calcification being more common in the free margin of the small intestine wall and in the mesentery. Catriona et al. [23] retrospectively analyzed abdominal CT findings for 27 cases diagnosed with EPS, and imaging manifestations such as peritoneal thickening, peritoneal calcification, intestinal wall thickening, abdominal separation and intestinal dilatation were analyzed by scoring in two groups of cases. The authors proposed that abdominal CT is a good diagnostic tool for EPS in suspicious cases. In our study, the manifestations of abdominal CT imaging with significant differences were scored, and the results showed that peritoneal dialysis might not be effective at a score greater than or equal to 2, with high sensitivity and specificity. Therefore, the establishment of a simple and practical scoring method based on the imaging features of abdominal CT can help physicians evaluate the efficacy of peritoneal dialysis in CAPD patients more objectively.

\section{Limitations}

One limitation is that this study had a single-center design, with a relatively limited sample size for the long-term CAPD group, as patients with CAPD for over 10 years are rare. Another limitation is the absence of biopsy evidence of the peritoneum. This is because PD patients rarely receive surgical treatment unless they develop very severe abdominal complications (e.g., intestinal obstruction).

In conclusion, there are certain characteristics of longterm CAPD on nonenhanced abdominal CT imaging. When extensive thickening of the parietal peritoneum, severe calcification of the parietal peritoneum or calcification of the mesentery are present, the effect of peritoneal dialysis may be poor.

Authors' contribution Conceptualization: XY; JG; Methodology: FZ, XD, CL, YP, JL, RX; Formal analysis and investigation: FZ, XD, CL, YP, JL, RX; Writing - original draft preparation: FZ, XD; Writingreview and editing: XY; JG; Supervision: XY; JG. All authors read and approved the final manuscript.

Funding No funding was received to assist with the preparation of this manuscript.

\section{Declarations}

Conflict of interest The authors have no relevant financial or non-financial interests to disclose.

Ethical approval All procedures performed in studies involving human participants were in accordance with the ethical standards of the insti- 
tutional research committee and with the 1964 Helsinki declaration and its later amendments or comparable ethical standards.

Informed consent Informed consent was not obtained for this retrospective review.

\section{References}

1. J.H. Hansson, S. Watnick, Update on Peritoneal Dialysis: Core Curriculum 2016, American journal of kidney diseases: the official journal of the National Kidney Foundation 67(1) (2016) 151-64. https://doi.org/https://doi.org/10.1053/j.ajkd.2015.06. 031

2. M. Wilkie, S. Davies, Peritoneal Dialysis in the time of COVID19, Peritoneal dialysis international: journal of the International Society for Peritoneal Dialysis 40(4) (2020) 357-358. https:// doi.org/https://doi.org/10.1177/0896860820921657

3. H.H. Yeter, E. Gok Oguz, O.F. Akcay, R. Karaer, E. Yasar, M. Duranay, M.D. Ayli, G. Guz, The reliability and success of peritoneal dialysis during the COVID-19 pandemic, Seminars in dialysis 34(2) (2021) 147-156. https://doi.org/https://doi.org/10.1111/sdi. 12940

4. K. Kawanishi, K. Honda, C. Hamada, Recommendations for pathological diagnosis on biopsy samples from peritoneal dialysis patients, Pleura and peritoneum 2(1) (2017) 3-15. https://doi. org/https://doi.org/10.1515/pp-2016-0028

5. H. Kinashi, Y. Ito, M. Mizuno, Y. Suzuki, T. Terabayashi, F. Nagura, R. Hattori, Y. Matsukawa, T. Mizuno, Y. Noda, H. Nishimura, R. Nishio, S. Maruyama, E. Imai, S. Matsuo, Y. Takei, TGF- $\beta 1$ promotes lymphangiogenesis during peritoneal fibrosis, Journal of the American Society of Nephrology: JASN 24(10) (2013) 1627-42. https://doi.org/https://doi.org/10.1681/asn.2012030226

6. R.T. Krediet, Ultrafiltration Failure Is a Reflection of Peritoneal Alterations in Patients Treated With Peritoneal Dialysis, Frontiers in physiology 9 (2018) 1815. https://doi.org/https://doi.org/10.3389/ fphys.2018.01815

7. A.T. van Diepen, S. van Esch, D.G. Struijk, R.T. Krediet, The first peritonitis episode alters the natural course of peritoneal membrane characteristics in peritoneal dialysis patients, Peritoneal dialysis international: journal of the International Society for Peritoneal Dialysis 35(3) (2015) 324-32. https://doi.org/https://doi.org/10. 3747/pdi.2014.00277

8. K. Kawanishi, K. Honda, M. Tsukada, H. Oda, K. Nitta, Neutral solution low in glucose degradation products is associated with less peritoneal fibrosis and vascular sclerosis in patients receiving peritoneal dialysis, Peritoneal dialysis international: journal of the International Society for Peritoneal Dialysis 33(3) (2013) 242-51. https://doi.org/https://doi.org/10.3747/pdi.2011.00270

9. C. George, K. Al-Zwae, S. Nair, J.E. Cast, Computed tomography appearances of sclerosing encapsulating peritonitis, Clinical radiology 62(8) (2007) 732-7. https://doi.org/https://doi.org/10.1016/j. crad.2007.01.022

10. B. Cakir, I. Kirbaş, B. Cevik, E.M. Ulu, A. Bayrak, M. Coşkun, Complications of continuous ambulatory peritoneal dialysis: evaluation with CT, Diagnostic and interventional radiology (Ankara, Turkey) 14(4) (2008) 212-20.

11. K. Dejima, H. Mitsuhashi, G. Yasuda, N. Hirawa, Y. Ikeda, S. Umemura, Localization and extent of peritoneal calcification in three uremic patients on continuous ambulatory peritoneal dialysis, Therapeutic apheresis and dialysis: official peer-reviewed journal of the International Society for Apheresis, the Japanese Society for Apheresis, the Japanese Society for Dialysis Therapy 12(5) (2008)
413-6. https://doi.org/https://doi.org/10.1111/j.1744-9987.2008. 00620.x

12. E.A. Brown, J. Bargman, W. van Biesen, M.Y. Chang, F.O. Finkelstein, H. Hurst, D.W. Johnson, H. Kawanishi, M. Lambie, T.P. de Moraes, J. Morelle, G. Woodrow, Length of Time on Peritoneal Dialysis and Encapsulating Peritoneal Sclerosis - Position Paper for ISPD: 2017 Update, Peritoneal dialysis international: journal of the International Society for Peritoneal Dialysis 37(4) (2017) 362-374. https://doi.org/https://doi.org/10.3747/pdi.2017.00018

13. V. Vizzardi, M. Sandrini, S. Zecchini, S. Ravera, L. Manili, G. Cancarini, Encapsulating peritoneal sclerosis in an Italian center: thirty year experience, Journal of nephrology 29(2) (2016) 259-267. https://doi.org/https://doi.org/10.1007/s40620-015-0241-x

14. S. Chugh, S. Chaudhry, T. Ryan, P.J.J.A.i.N. Margetts, Peritoneal Membrane Injury and Peritoneal Dialysis, 2014 (2014).

15. R.M. Tarzi, A. Lim, S. Moser, S. Ahmad, A. George, G. Balasubramaniam, E.J. Clutterbuck, W. Gedroyc, E.A. Brown, Assessing the validity of an abdominal CT scoring system in the diagnosis of encapsulating peritoneal sclerosis, Clinical journal of the American Society of Nephrology: CJASN 3(6) (2008) 1702-10. https://doi. org/https://doi.org/10.2215/cjn.01820408

16. H. Inoshita, T. Gohda, H. Io, K. Kaneko, C. Hamada, S. Horikoshi, Y. Tomino, Improvement of peritoneal calcification after parathyroidectomy in a peritoneal dialysis patient, Clinical nephrology 69(1) (2008) 58-62. https://doi.org/https://doi.org/10.5414/cnp69 058

17. N. Di Paolo, G. Sacchi, P. Lorenzoni, E. Sansoni, E. Gaggiotti, Ossification of the peritoneal membrane, Peritoneal dialysis international: journal of the International Society for Peritoneal Dialysis 24(5) (2004) 471-7.

18. A.Y. Wang, Calcium balance and negative impact of calcium load in peritoneal dialysis patients, Peritoneal dialysis international: journal of the International Society for Peritoneal Dialysis 34(4) (2014) 345-52. https://doi.org/https://doi.org/10.3747/pdi.2013.00177

19. K.N. Lai, J.C. Leung, Inflammation in peritoneal dialysis, Nephron. Clinical practice 116(1) (2010) c11-8. https://doi.org/https://doi.org/ $10.1159 / 000314544$

20. T.C. Lee, J.Y. Yang, H.P. Wang, T.J. Tsai, Y. Yang, Peritoneal thickening is not inevitable in long-term peritoneal dialysis and is associated with peritoneal transport characteristics: a two-centre sonographic study, Nephrology, dialysis, transplantation: official publication of the European Dialysis and Transplant Association - European Renal Association 23(3) (2008) 1005-10. https://doi. org/https://doi.org/10.1093/ndt/gfm726

21. A. Vlijm, S.S. Phoa, M. Noordzij, A.M. Spijkerboer, J. van Schuppen, J. Stoker, D.G. Struijk, R.T. Krediet, Are peritoneal calcifications in long-term peritoneal dialysis related to aortic calcifications and disturbances in mineral metabolism?, Nephrology, dialysis, transplantation : official publication of the European Dialysis and Transplant Association - European Renal Association 26(1) (2011) 304-8. https://doi.org/https://doi.org/10.1093/ndt/gfq374

22. Yang J, Xiao F, Chen J, Hou ShH, Wang Y, Zhang WG, Gong WJ, Yu Y, Cai MY, He YN, The changes of abdominal CT image and their influence factors in peritoneal dialysis related peritoneal injury. Chin J Blood Purif 18(12) (2019) 809-821

23. C. Goodlad, R. Tarzi, W. Gedroyc, A. Lim, S. Moser, E.A. Brown, Screening for encapsulating peritoneal sclerosis in patients on peritoneal dialysis: role of CT scanning, Nephrology, dialysis, transplantation: official publication of the European Dialysis and Transplant Association - European Renal Association 26(4) (2011) 1374-9. https://doi.org/https://doi.org/10.1093/ndt/gfq533

Publisher's Note Springer Nature remains neutral with regard to jurisdictional claims in published maps and institutional affiliations. 